\title{
Long-term variation of galactic cosmic ray intensity observed with the Nagoya multidirectional muon detector
}

\author{
K. Munakata* \\ Shinshu University, Nagano, Japan \\ E-mail: kmuna00eshinshu-u.ac.jp \\ C. Kato \\ Shinshu University, Nagano, Japan \\ E-mail: ckato@shinshu-u.ac.jp
}

\section{R. R. S. Mendonça}

State Key Laboratory of Space Weather, National Space Science Center, Chinese Academy of Sciences, Beijing, China

National Institute for Space Research, São José dos Campos São Paulo, Brazil

E-mail: rafael.mendoncalinpe.br

\section{Tokumaru}

ISEE, Nagoya University, Nagoya, Aichi, JAPAN

E-mail: tokumarulisee.nagoya-u.ac.jp

\begin{abstract}
We analyze long-term variations of galactic cosmic ray intensity observed with the Nagoya multidirectional muon detector during a period between 1970 and 2016 which covers four solar activity cycles. We particularly analyze the rigidity spectrum of the modulation in 16-107 GV rigidity range by using the data recorded in 17 directional channels of Nagoya muon detector together with 6 neutron monitor data. This paper reports very preliminary results of our analysis.
\end{abstract}

36th International Cosmic Ray Conference -ICRC2019-

July 24th - August 1st, 2019

Madison, WI, U.S.A.

\footnotetext{
${ }^{*}$ Speaker.
} 


\section{Introduction}

We have recently developed a method of correcting the atmospheric temperature effect on the ground level muon count rate by analyzing the data collected with the GMDN (Global Muon Detector Network), which is a global network consisting of four multidirectional muon detectors [1]2]. This is a significant step, because it makes possible for the first time to analyze the long-term variation (such as 11-year and 22-year variations) of cosmic ray density (i.e. isotropic intensity) in 50-100 GV rigidity range, which has been possible only for cosmic rays around $\sim 10 \mathrm{GV}$ observed by neutron monitors. In this report, we analyze the long-term variation observed with Nagoya multidirectional muon detector (MD) during a period between 1970 and 2016 which covers four solar activity cycles. We particularly analyze the rigidity dependence of variations in $\sim 10-100 \mathrm{GV}$ range by using the data recorded in 17 directional channels of Nagoya-MD together with neutron monitor (NM) data.

\section{Data used for analysis}

We use muon count rates recorded in 47 years between 1970 and 2016 in 17 directional channels of Nagoya-MD. These count rates are corrected for the atmospheric effects (pressure and temperature effects) and for the instrumental discontinuities in the count rate. For the correction of the temperature effect, we use the mass-weighted atmospheric temperature calculated from the meteorological data provided by the Global Data Assimilation System (GDAS) maintained by the NOAA's Air Resources Laboratory (ARL). It is verified that the large amplitude seasonal variation due to the temperature effect is successfully removed from the observed count rate by our correction method [1, 2].

We also use the pressure corrected data recored by 6 NMs at McMurdo, Thule, Rome, Tokyo, Tibet and Doi Inthanon during the same period. By using Nagoya-MD data together with these NM data, we can analyze the intensity variation in a wide range of the median primary rigidity $\left(P_{m}\right)$ between 16 and $107 \mathrm{GV}$. We estimate $P_{m}$ by using the so-called Nagashima's response function for NMs [3] and Murakami's response function for Nagoya-MD [4].

\section{Analysis and Result}

The lower panel of Figure 1 shows long-term variations of muon count rate recorded in three directional channels of Nagoya-MD and corrected for the atmospheric effects (bottom three colored curves), together with variations recorded by two NMs (top black and gray curves). The variation in each curve is multiplied by an indicated factor to plot all curves on the same vertical scale and is shifted from each other to avoid overlapping. It is seen that all variations recorded by Nagoya-MD and NMs show a common feature of the intensity varying in a clear anti-correlation with the solar activity represented by the sunspot number and the heliospheric current sheet tilt-angle in the upper panel. It is also seen that the variation amplitude is approximately proportional to $1 / P_{m}$ decreasing with increasing $P_{m}$. Unknown "seasonal variation" within one year is also notable particularly 
in Nagoya-MD data, but we suspect the possibility that this is due to insufficient correction of Nagoya-MD data for atmospheric effect because we see similar variations also in NM data. The origin of this seasonal variation will be analyzed in detail in separate paper.

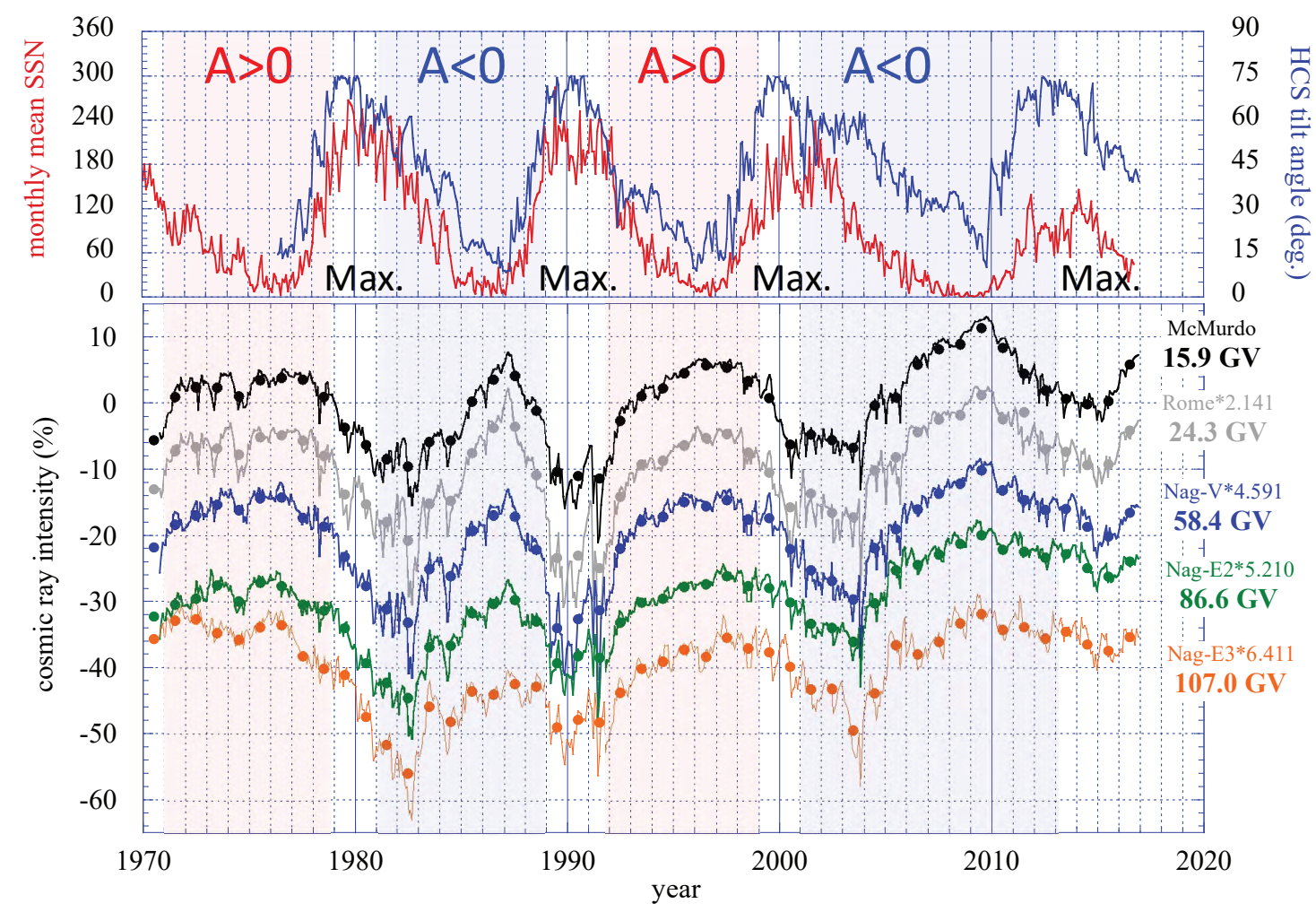

Figure 1: The long-term variation of cosmic ray intensity observed with NMs and Nagoya-MD in a period between 1970 and 2016. Upper panel displays temporal variations of monthly mean sunspot number (red curve) on the left vertical axis and Carrington rotation average of the heliospheric current sheet tilt-angle (blue curve) on the right vertical axis. The polarity $(\mathrm{A}>0$ or $\mathrm{A}<0)$ of the solar magnetic dipole and the solar activity maximum and minimum (Max. and Min.) are indicated in the figure. The lower panel shows cosmic ray intensity variations in \% observed by two NMs (top black and gray curves) and by Nagoya-MD (bottom three colored curves). Solid circles in each curve show yearly mean intensity, while thin curves with the same color show the Carrington rotation mean intensity. Each curve in the lower panel is multiplied by a factor indicated in the figure to plot all curves on the same vertical scale and is shifted from each other to avoid overlapping.

An interesting feature of variations in Figure 1 is seen in the difference between intensities in $\mathrm{A}>0$ solar minimum around 1976 and in $\mathrm{A}<0$ solar minimum around 1987. The intensity by NMs is higher in $\mathrm{A}<0$ solar minimum than in $\mathrm{A}>0$ solar minimum, but this correlation with the solar magnetic dipole polarity $(\mathrm{A}>0$ or $\mathrm{A}<0)$ tends to reverse in high $P_{m}$ intensity monitored by the Nagoya-MD, implying that the rigidity spectrum of the solar modulation is also time dependent. To analyze the temporal variation of the rigidity spectrum, we first convert the yearly mean intensity $\left(I_{i}^{\text {obs }}(t)\right)$ observed by the $i$-th component of total 23 components (6 NMs plus 17 directional channels of Nagoya-MD) in a year $t$ to the intensity $\left(\Delta I_{i}^{\mathrm{obs}}(t)\right)$ relative to $I_{i}^{\mathrm{obs}}(2009)$ in 2009 when 
the McMurdo NM recorded the highest count rate in 1970-2016, as

$$
\Delta I_{i}^{\mathrm{obs}}(t)=1-I_{i}^{\mathrm{obs}}(t) / I_{i}^{\mathrm{obs}}(2009)
$$

By assuming a simple power-law spectrum for the modulation, we model the relative intensity, as

$$
\Delta I_{i}^{\mathrm{fit}}(t)=1-\int_{P_{c i}}^{\infty} c(t)(p / 10 \mathrm{GV})^{\gamma(t)} R_{i}(p) d p / \int_{P_{c i}}^{\infty} R_{i}(p) d p
$$

where $p$ is the rigidity of primary cosmic rays in $\mathrm{GV}, R_{i}(p)$ and $P_{c i}$ are the response function and the geomagnetic cut-off rigidity for the $i$-th component, respectively [3, 4]. We obtain the best-fit parameters $c(t)$ and $\gamma(t)$ in a year $t$ minimizing,

$$
S(t)=\sum_{i}\left(\Delta I_{i}^{\mathrm{obs}}(t)-\Delta I_{i}^{\mathrm{fit}}(t)\right)^{2} / \sigma_{i}^{2}
$$

where $\sigma_{i}$ is the error of $\Delta I_{i}^{\text {obs }}(t)$ deduced from the dispersion of hourly count rates in the corresponding year.

The middle panel of Figure 2 shows the best-fit $\gamma(t)$ as a function of year, while the bottom panel shows the bset-fit $1-\Delta I_{i}^{\mathrm{fit}}(t)$ (i.e. the second term on the right side of 3.2 representing the modulation magnitude at $P_{m}=10 \mathrm{GV}$ and $100 \mathrm{GV}$ by blue and red curves, respectively. Note that the vertical axis of this panel is reversed for representing the intensity variation instead of the modulation magnitude. It is seen that the modulation spectrum is significantly harder in 1987 than in 1976, resulting in the observed feature in Figure 1 that the intensity at lower (higher) $P_{m}$ is higher (lower) in $\mathrm{A}<0$ solar minimum than in $\mathrm{A}>0$ solar minimum.

\section{Discussion}

By using yearly mean intensities observed by 17 directional channels of the Nagoya-MD and by $6 \mathrm{NMs}$, we analyzed the rigidity spectrum of the GCR intensity modulation in a range of the median primary rigidity between 16 and $107 \mathrm{GV}$. It was shown that the modulation spectrum is significantly harder in 1987 than in 1976, resulting in the observed intensity at lower (higher) $P_{m}$ being higher (lower) in $\mathrm{A}<0$ solar minimum than in $\mathrm{A}>0$ solar minimum. This implies that there is a "cross-over" of two spectra in 1976 and 1987 at around $30 \mathrm{GV}$, in addition to that at $\sim 7$ GV reported from the latitude survey using NMs [5]. Such a pair of two cross-overs at low and high rigidities has been actually predicted by the drift model calculation [6]. While the long-term observation with Nagoya-MD is very important for accurately deducing the modulation spectrum and its temporal variation, our analysis still has to be improved to reduce possible systematic errors arising from incomplete data corrections.

\section{Acknowledgment}

This work is supported in part by the joint research programs of the the Institute for SpaceEarth Environmental Research (ISEE), Nagoya University and the Institute of Cosmic Ray Research (ICRR), University of Tokyo. The observation with the Nagoya multidirectional muon 


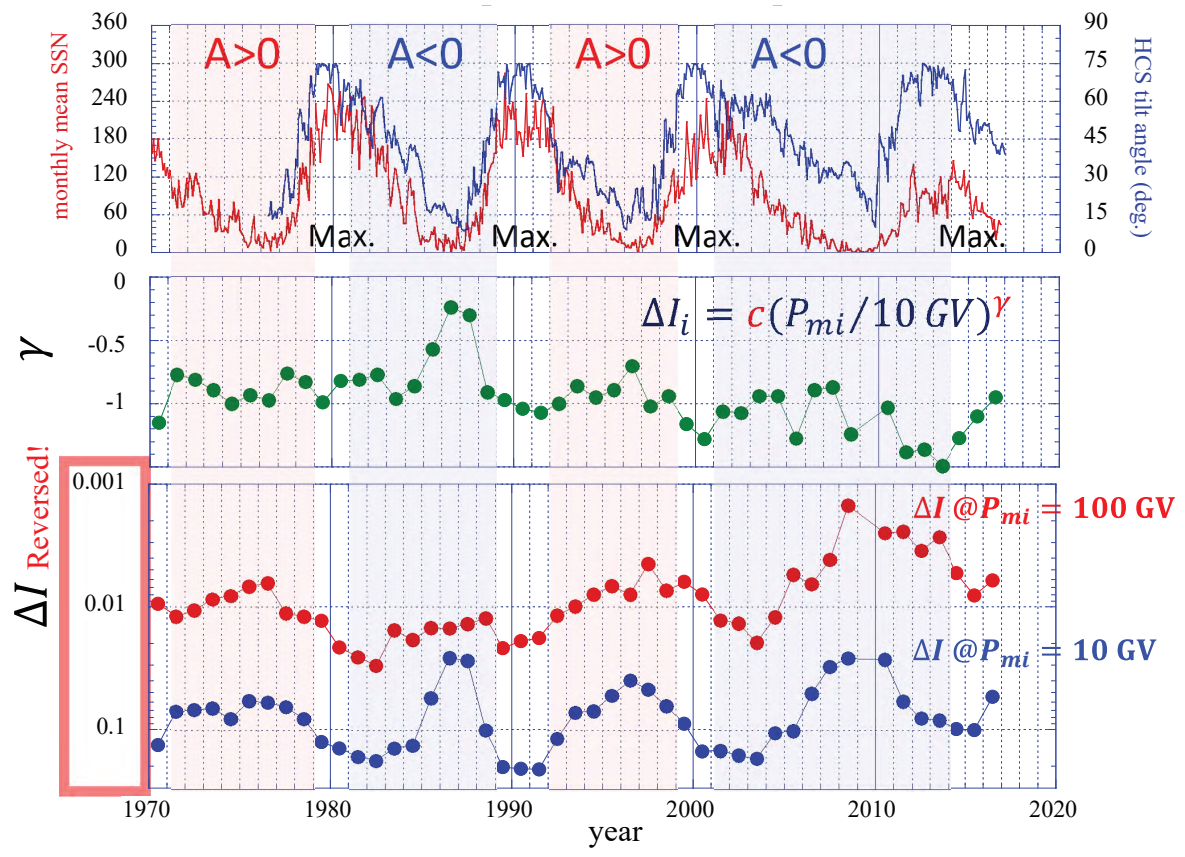

Figure 2: The temporal variation of the rigidity spectrum parameters obtained from best-fitting the powerlaw spectrum to the observed yearly mean cosmic ray intensity (see text). Middle panel shows the power-law index $\gamma(t)$, while bottom panel displays magnitude of the modulation at $10 \mathrm{GV}$ (blue curve) and at $100 \mathrm{GV}$ (red curve), each as a function of year. Note that the vertical axis of the bottom panel is reversed to represent variation of the intensity instead of the magnitude of modulation. Top panel displays temporal variations of monthly mean sunspot number (red curve) and Carrington rotation average of the heliospheric current sheet tilt-angle (blue curve) in the same manner as Figure 1.

detector is supported by the ISEE, Nagoya University. The Bartol Re-search Institute neutron monitor program, which operates Thule and McMurdo neutron monitors, is supported by National Science Foundation grant ATM-0000315. The SVIRCO NM at Rome is supported by INAF/IAPSUNIRoma3 COLLABORATION. Neutron monitor data from Doi Inthanon were provided by courtesy of the Princess Sirindhorn Neutron Monitor Program. We thank the World Data Center for Cosmic Rays of the ISEE, Nagoya University, for providing NM data. We obtained the Wilcox Solar Observatory data via the Web site http://wso.stanford.edu at 2014:03:19 01:10:41 PDT courtesy of J. T. Hoeksema. The Wilcox Solar Observatory is currently supported by NASA. R.R.S.M. thanks the China-Brazil Joint Laboratory for Space Weather.

\section{References}

[1] R. R. S. Mendonça et al., Proceedings of the International Astronomical Union, 12, 130, 2016.

[2] R. R. S. Mendonça et al., Astrophys. J., 830, 88, 2016.

[3] K. Nagashima et al., Il Nuovo Cim., 12C, N2, 173, 1989.

[4] K. Murakami et al., Il Nuovo Cim, 2C, N5, 635, 1987.

[5] H. Moraal et al., J. of Geophys. Res., 94, A2, 1469, 1989. 
[6] J. P. L. Reinecke and M. S. Potgieter, J. of Geophys. Res., 99, A8, 14761, 1994. 